A Tapping-Mode Tuning Fork with a Short Fibre Probe Sensor for a Near-Field Scanning Optical Microscope

This article has been downloaded from IOPscience. Please scroll down to see the full text article. 2002 Chinese Phys. Lett. 191268

(http://iopscience.iop.org/0256-307X/19/9/315)

The Table of Contents and more related content is available

Download details:

IP Address: 140.112.113.225

The article was downloaded on 22/07/2009 at 08:28

Please note that terms and conditions apply. 


\title{
A Tapping-Mode Tuning Fork with a Short Fibre Probe Sensor for a Near-Field Scanning Optical Microscope*
}

\author{
WANG Pei(王沛) $)^{1}$, LU Yong-Hua(鲁拥华 $)^{1}$, ZHANG Jiang-Ying(章江英 $)^{1}$, MING Hai(明海 $)^{1}$, \\ XIE Jian-Ping(谢建平 $)^{1}$, HUANG Chien-Wen(黄建文 $)^{2}$, KAO Tsung-Sheng(高宗圣 $)^{2}$, \\ TSAI Din-Ping(蔡定平 $)^{2}$ \\ ${ }^{1}$ Department of Physics, University of Science and Technology of China, Hefei 230026 \\ ${ }^{2}$ Department of Physics, National Taiwan University, Taipei 10617
}

(Received 20 May 2002)

\begin{abstract}
We report on a tapping-mode tuning fork with a short fibre probe sensor for a near-field scanning optical microscope. The method demonstrates how to fabricate the short fibre probe. This tapping-mode tuning fork with a short fibre probe can provide stable and high $Q$ at the tapping frequency of the tuning fork, and can give high-quality near-field scanning optical microscope and atomic force microscope images of samples. We present the results of using the tapping-mode tuning fork with a short fibre probe sensor for a near-field scanning optical microscope performed on an eight-channel silica waveguide.
\end{abstract}

PACS: 07.79.FC

The near-field scanning optical microscope (NSOM) is an instrument that can provide a detailed view of the optical fields and topography of materials with high spatial resolution beyond the diffraction limits. ${ }^{[1]}$ NSOMs are widely used in nanometre technology, biological technology, high-density optical storage and measurements of local spectrum, and photonic devices, etc. ${ }^{[2-4]}$ A typical NSOM uses a fibre probe to form a point light source or collector, and uses shear force or a tapping-mode tuning fork as feedback to keep the probe in proximity with the sample surface. In order to make an NSOM work well, the tuning fork with a fibre probe must own stable and high $Q$ at the tapping frequency of the tuning fork. ${ }^{[5]}$ For the tapping-mode NSOM, the tuning fork with the fibre probe was forced to oscillate by the bimorph. This can be described by the equation of the classical oscillator as ${ }^{[6]}$

$$
m\left(\ddot{x}+\gamma \dot{x}+\omega_{0}^{2}\right)=F_{0} \mathrm{e}^{\mathrm{i} \omega t},
$$

where $m$ is the mass of the oscillator (here it is the mass of vibrated tine and the fibre probe), $F_{0} \mathrm{e}^{\mathrm{i} \omega t}$ is the driving force, $\omega_{0}$ is the eigenfrequency of the oscillator, which is inversely proportion to $\sqrt{m}, x$ is the displacement, and $\gamma$ is the damping coefficient. The solution of Eq. (1) reads

$$
x=x_{0} \mathrm{e}^{\mathrm{i} \omega t}=\frac{F_{0}}{m} \cdot \frac{-1}{\omega^{2}-\mathrm{i} \gamma \omega-\omega_{0}^{2}} .
$$

The $Q$ value of the oscillation is written as

$$
Q=\frac{\omega_{0}}{\gamma}
$$

Thus, the fibre probe with a long tail is too heavy for the tuning fork to own a high $Q$ value frequency response. The bad frequency response makes it hard to keep the probe in the exact vicinity of the sample.

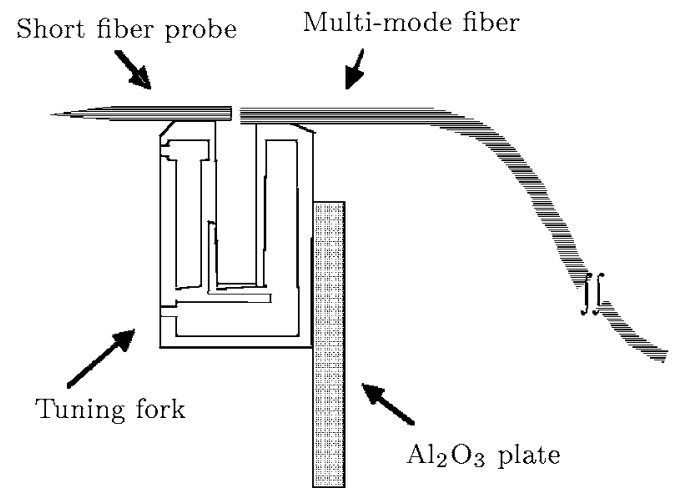

Fig. 1. Schematic construction of the tapping-mode tuning fork with a short fibre probe.

In this Letter, in order to solve this problem, we report on a tapping-mode tuning fork with a short fibre probe sensor for a NSOM. The method demonstrates how to fabricate the short fibre probe. The frequency response around the resonant peak is measured for long probes and, in contrast, for short probes. Furthermore, the optical intensity distributions at the end-face of an eight-channel silica waveguide are measured by using the tapping-mode tuning fork with a short fibre probe sensor for the NSOM, giving highquality NSOM and atomic force microscope (AFM) images of the sample.

A schematic diagram of our tapping-mode tuning fork with a short fibre probe set-up is shown in Fig. 1. The tuning fork is commercially available with a resonance quality $Q$ of 80000 and a resonance frequency

\footnotetext{
* Sponsored by the National Research Fund for Fundamental Key 973-Project under Grant No G1999033002 and the National Natural Science Foundation of China under Grant No 19974042.

(C) 2002 Chinese Physical Society and IOP Publishing Ltd
} 

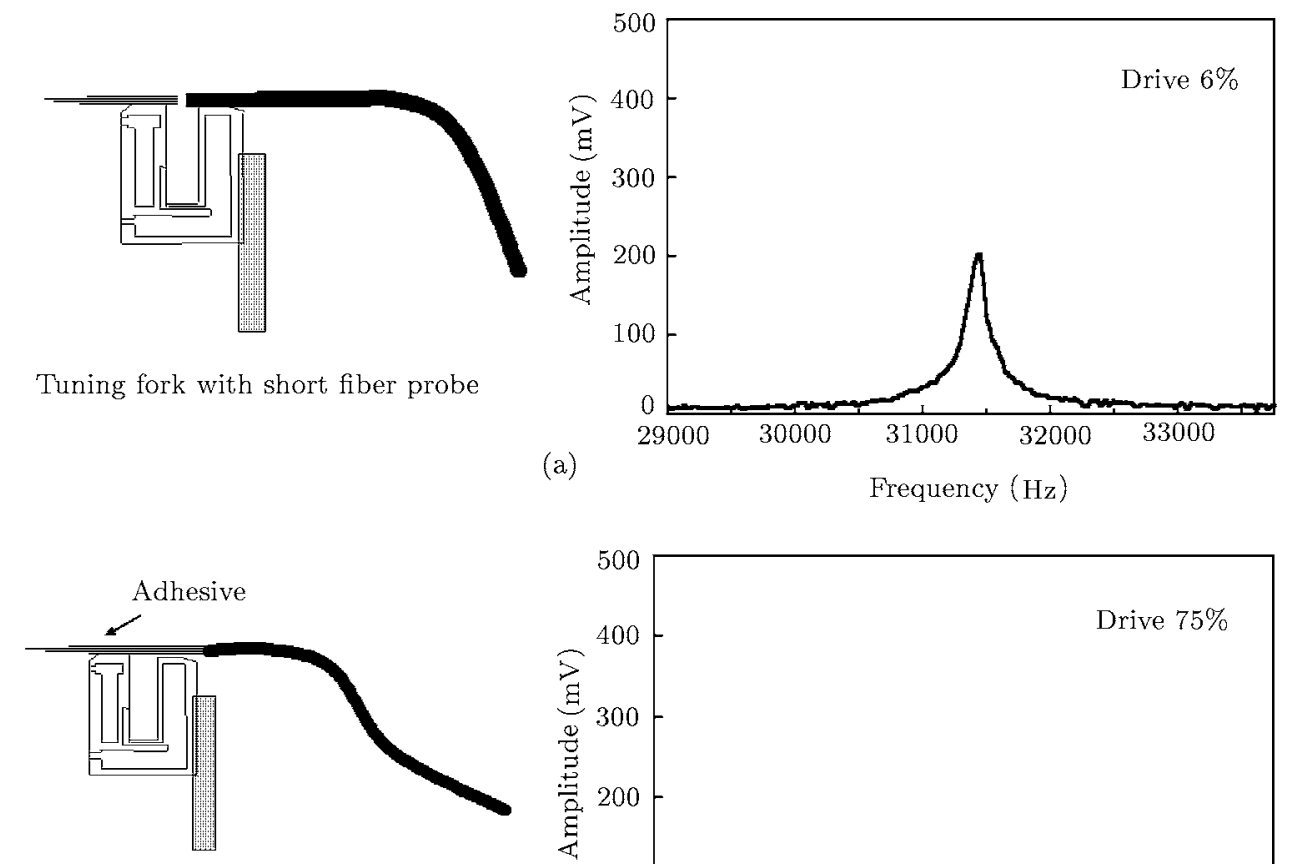

Tuning fork with long tail fiber probe

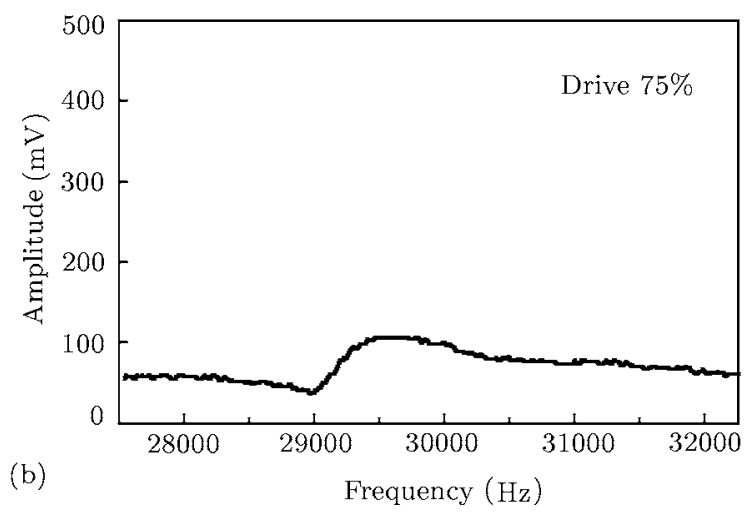

Fig. 2. Tapping frequency and amplitude of a tuning fork with (a) a short fibre probe and (b) a long-tail fibre probe.

fibre probe

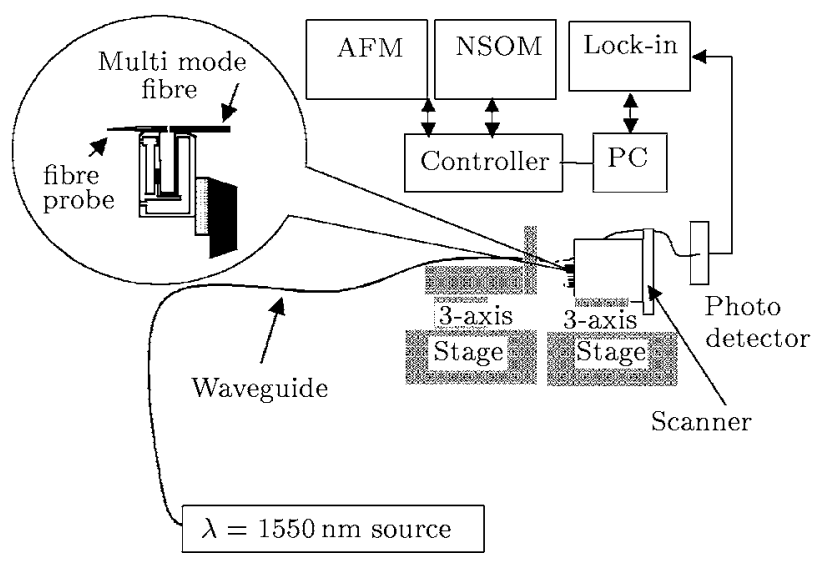

Fig. 3. Schematic diagram of the dual-mode NSOM.

of $32.768 \mathrm{kHz}$. One tine of the tuning fork is fixed on an $\mathrm{Al}_{2} \mathrm{O}_{3}$ disc by cyanoacrylate adhesive (superglue), with the fork protruding $(\sim 3.5 \mathrm{~mm})$ from the edge of the disc. A short near-field optical fibre probe pulled by commercially available puller, and subsequently coated with a thin layer of aluminium, is attached by epoxy at the end of the opposite tine of the tuning fork, with the fibre tip sticking out by about $1 \mathrm{~mm}$. Then, we glue a multi-mode fibre on the edge of the tine fixed on the disc to receive the light signal from the short probe. There is a very small split between the short fibre probe and the multi-mode fi- bre to keep them from contacting each other. All these fabrications were performed under optical microscopy.

Figures 2(a) and 2(b) show the tapping frequency and amplitude of a tapping-mode tuning fork with a short fibre probe and with a long fibre probe, respectively. The long fibre probe is as long as the multimode fibre. It is obvious that the tapping-mode tuning fork with a short fibre probe can provide stable and high $Q$ (around 150) at the tapping frequency of the tuning fork, although the resonance peak shifts from $32.768 \mathrm{kHz}$ for the free fork to $31.45 \mathrm{kHz}$ for the fork with a short fibre probe; this frequency shift is predicted from Eq. (2). The frequency response of the long fibre probe has $Q$ of only 29 and a large resonance frequency shift (from $32.768 \mathrm{kHz}$ to $29.60 \mathrm{kHz}$ ). The experiment also shows that the resonance frequency and amplitude of the tapping-mode tuning fork with a short fibre probe do not change with the increasing length of the multi-mode fibre. This is because the short fibre probe and the multi-mode fibre are apart, and the multi-mode fibre does not affect the frequency response of the tapping-mode tuning fork with a short fibre probe. The greater frequency shift in Fig. 2(b) than in Fig. 2(a) indicates that the long fibre tail adds the mass of the oscillator, which decreases the $Q$ value. In addition, the long fibre tail also increases the $\gamma$ value; it contributes more to the decrease of $Q$. It can be further verified that the frequency response of the tapping-mode tuning fork with a long fibre probe 

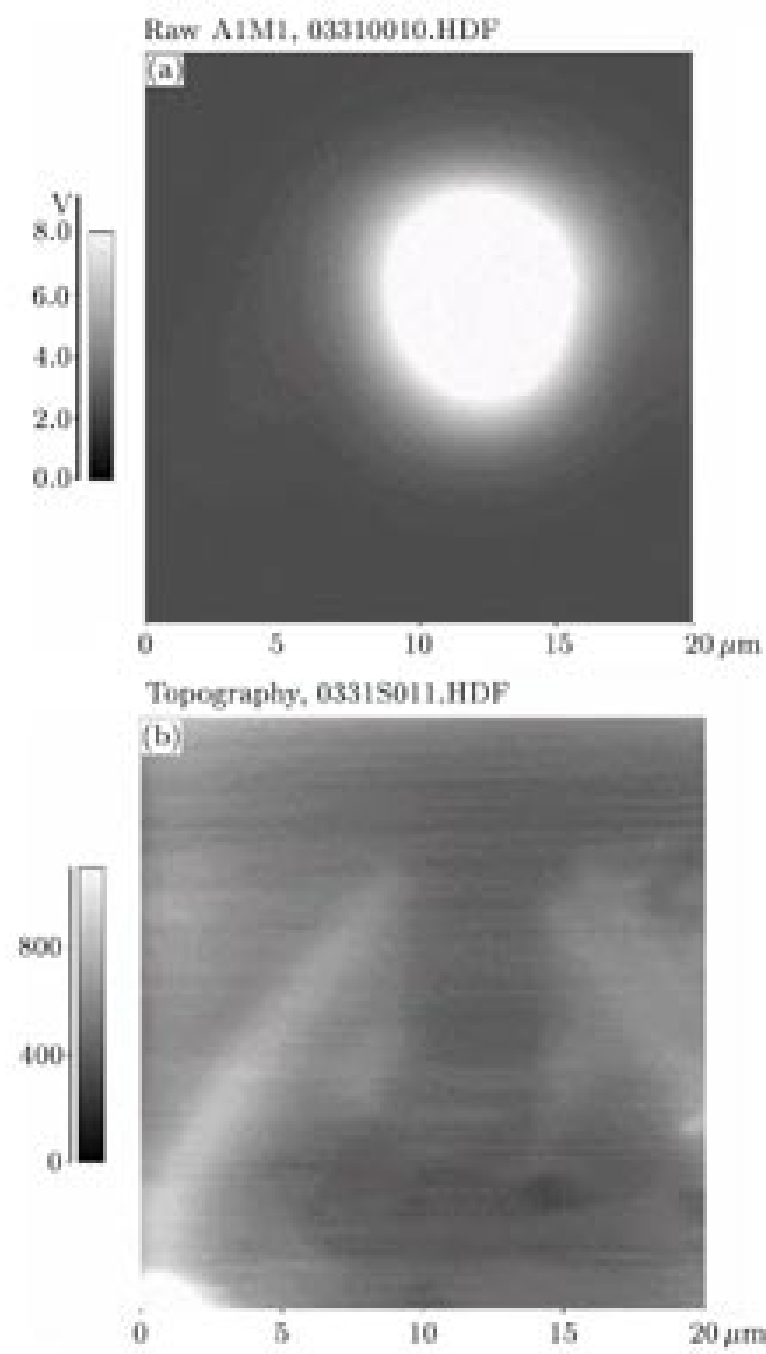

Fig. 4. (a) NSOM and (b) AFM images of the end-face of the channel waveguide with a $1550 \mathrm{~nm}$ light source injected by using the short fibre probe.

becomes worse, even disappearing, with the increasing fibre length.

We have subsequently used this new type of tapping-mode tuning fork with a short fibre probe to measure the NSOM and AFM images at the end-face of the eight-channel silica waveguide. The schematic diagram for these measurements is shown in Fig. 3. The silica channel waveguide is made by the semiconductor processing, injected with a $\lambda=1550 \mathrm{~nm}$ infrared source. Figure 4 shows the measured NSOM and AFM images of the silica waveguide with the same scan area size. All these images show that a tappingmode tuning fork with a short fibre probe can easily provide high-quality NSOM and AFM images of samples.

In summary, we have successfully developed a tapping-mode tuning fork with a short fibre probe sensor for an NSOM. The advantages of this short fibre probe are shown and explained both theoretically and experimentally. This type of tapping-mode tuning fork with a short fibre probe can provide more stable and higher $Q$ at the tapping frequency of the tuning fork than the traditional tapping-mode $\mathrm{NSOM}^{[5]}$ with a long fibre robe. Therefore, high-quality NSOM and AFM images of samples can easily be achieved using the tapping-mode NSOM described here.

\section{References}

[1] Betzig E and Trautman J K 1992 Science 257189

[2] Tsai D P and Li W K 1997 J. Vac. Sci. Technol. A 15 1427

[3] Tsai D P, Yang Ch W, Lo S Z, Jackson H E 1999 Appl. Phys. Lett. 751039

[4] Lu N H, Tsai D P, Chang C S and Tsong T T 1999 Appl. Phys. Lett. $\mathbf{7 4} 2746$

[5] Tsai D P and Lu Y Y 1998 Appl. Phys. Lett. 732724

[6] Tamaru H and Miyano K 1997 Japan. J. Appl. Phys. 36 L821 